Artigo Original

\title{
A utilização do critério da Organização Mundial de Saúde para classificação do estado nutricional em crianças
}

\author{
Valter Cordeiro Barbosa Filho ${ }^{1,3}$ \\ Teresa Maria Bianchini de Quadros ${ }^{2}$ \\ Evanice Avelino de Souza ${ }^{1}$ \\ Alex Pinheiro Gordia ${ }^{2}$ \\ Wagner de Campos ${ }^{3}$ \\ ${ }^{1}$ Grupo de pesquisa em Tratamento e Prevenção da Obesidade em Adolescentes, \\ Universidade Federal do Ceará, Fortaleza, CE, Brasil \\ ${ }^{2}$ Universidade Federal do Recôncavo da Bahia, Cruz das Almas, BA, Brasil \\ ${ }^{3}$ Programa de Pós-graduação em Educação Física da Universidade Federal do Paraná, \\ Curitiba, PR, Brasil
}

\begin{abstract}
Resumo: Este estudo analisou a concordância do critério da Organização Mundial de Saúde (OMS, 2007) com os critérios de Kuczmarski et al. (2002), Cole et al. (2000, 2007) e Conde e Monteiro (2006) para classificação do estado nutricional em crianças. Estudo transversal realizado com 619 escolares (331 meninas) de 6 e 7 anos de idade, da cidade de Fortaleza, Ceará. Aferiu-se estatura e massa corporal para cálculo do índice de massa corporal. O índice Kappa (k) foi utilizado para analisar a concordância entre os critérios, considerando $p<0,05$. As concordâncias do critério da OMS (2007) foram significativas $(p<0,001)$, sendo muito boas $(k=0,82)$ com Kuczmarski et al. $(2002)$ e Cole et al. $(2000,2007)$, e boa $(k=0,68)$ para Conde e Monteiro (2006). Em conclusão, sugere-se cautela na utilização do critério da OMS, assim como dos demais, para avaliação do estado nutricional em crianças brasileiras devido às diferenças étnicas e metodológicas.
\end{abstract}

Palavras-chave: Estado nutricional. Crianças. Diagnóstico. Desnutrição. Excesso de peso.

\section{Use of World Health Organization criteria for nutritional status classification in children}

\begin{abstract}
This study analyzed the concordance of the World Health Organization criteria (WHO, 2007) with Kuczmarski et al. (2002), Cole et al. $(2000,2007)$ and Conde and Monteiro (2006) criteria used for nutritional status classification in children. This is a cross-sectional study, with 619 students (331 girls) from 6 and 7 years old, from the city of Fortaleza, Ceará, Brazil. Body mass and stature were measured to calculate the body mass index. The Kappa index (k) was used to analyze the concordance between the criteria, considering $p<0.05$. The concordances of the WHO (2007) criteria with the other three criteria were all significant $(p<0.001)$ : very good $(k=0.82)$ with Kuczmarski et al. $(2002)$ and Cole et al. $(2000,2007)$; good $(k=0.68)$ with Conde and Monteiro (2006). In conclusion, the World Health Organization criteria should be use with caution, as well as the others, to assess the nutritional status of Brazilian children due to ethnic and methodological differences.
\end{abstract}

Key Words: Nutritional status. Children. Diagnosis. Malnutrition. Overweight.

\section{Introdução}

A população brasileira passa por uma transição nutricional caracterizada pela diminuição da desnutrição (MONTEIRO et al., 2009), principalmente nos grandes centros urbanos, e pelo aumento da obesidade (MONTEIRO; CONDE; POPKIN, 2004; BATISTA FILHO; RISSIN, 2003). Desta forma, a obesidade se tornou um dos principais problemas de saúde pública (WANG; MONTEIRO; POPKIN, 2002), visto que se inicia cada vez mais cedo e está relacionada com fatores de risco para doenças cardiovasculares e síndrome metabólica
(JANSSEN et al., 2005; KATZMARZYK et al., 2003).

A Organização Mundial de Saúde (OMS, 1995) recomenda a utilização do Índice de Massa Corporal (IMC) para o diagnóstico do estado nutricional de grupos populacionais por ser um método não-invasivo, válido e de baixo custo (NIHISER et al., 2007). Em adultos, a OMS propôs os pontos de corte do IMC de $25 \mathrm{~kg} \cdot \mathrm{m}^{-2} \mathrm{e}$ $30 \mathrm{~kg} \cdot \mathrm{m}^{-2}$ para o diagnóstico de sobrepeso e obesidade, respectivamente (OMS, 1998). No entanto, como o IMC muda substancialmente com o avanço da idade, estes pontos de corte não são adequados para classificação do estado 
nutricional em crianças e adolescentes (MAYNARD et al., 2001; COLE; FREEMAN; PREECE, 1995). A partir disso, diversos critérios de classificação do IMC, de acordo com o sexo e idade, foram propostos para crianças e adolescentes (MUST et al., 1991; COLE et al., 2000; KUCZMARSKI et al., 2002; CONDE; MONTEIRO, 2006). No entanto, ainda não há um critério de classificação do estado nutricional em crianças e adolescentes universalmente aceito (NEOVIUS et al., 2004).

Em 2007, a OMS propôs um critério de classificação do estado nutricional para crianças e adolescentes entre 5 e 19 anos de idade, com a finalidade de monitorar e acompanhar infantojuvenis de vários países (BUTTE; GARZA; ONIS, 2007). Esse recente critério foi desenvolvido a partir dos dados americanos do National Center for Health Statistics (1977), com um reprocessamento dos dados para se adequar ao padrão de crescimento das crianças menores de cinco anos de idade, identificado em estudo multicêntrico realizado pela OMS em 2006, e aos pontos de corte definidos para a classificação do estado nutricional em adultos (ONIS et al., 2007).

Devido à sua recente publicação, o critério da OMS (2007) ainda é pouco utilizado para diagnósticos nutricionais em crianças e pouco se sabe sobre a concordância desse critério com outros critérios de classificação do estado nutricional utilizados na literatura brasileira. Não obstante, a utilização de diferentes critérios pode gerar divergências no diagnóstico do estado nutricional em crianças, dificultando a comparação entre estudos. Neste sentido, o objetivo deste estudo foi analisar o estado nutricional de crianças de seis e sete anos de idade e verificar a concordância do critério da OMS (2007) com os critérios propostos por Kuczmarski et al. (2002), Cole et al. (2000, 2007) e Conde e Monteiro (2006).

\section{Materiais e Métodos}

Este estudo apresentou delineamento transversal e foi realizado no mês de maio de 2007, com uma amostra representativa dos escolares de seis e sete anos de idade regularmente matriculados nas escolas públicas e particulares da cidade de Fortaleza, Ceará. O Termo de Consentimento Livre e Esclarecido (TCLE) foi assinado pelos pais ou responsáveis de cada escolar incluído na investigação, assegurando-se o anonimato das informações. 0 estudo foi aprovado pelo Comitê de Ética em Pesquisa da Universidade Federal do Ceará (protocolo $n^{\circ}$ 075/07).

A população do estudo foi composta por 70.000 crianças de seis e sete anos de idade que frequentavam as escolas públicas e particulares nas seis regiões do município de Fortaleza, de acordo com o levantamento do Instituto Brasileiro de Geografia e Estatística (IBGE, 2005). Calculou-se uma amostra aleatória simples, considerando a maior prevalência de excesso de peso $(15,0 \%)$ observada em crianças do município (PEREIRA; LIMA; MARTINS, 2004), com nível de confiança de $95 \%$ e erro amostral de três pontos percentuais. Após o acréscimo de uma margem de segurança de $10 \%$ para eventuais perdas amostrais, foi obtido o tamanho final da amostra para o estudo $(n=618)$.

Tabela 1. Distribuição dos escolares de acordo com o sexo e idade.

\begin{tabular}{lcccccc}
\hline Idade & \multicolumn{2}{c}{ Masculino } & \multicolumn{2}{c}{ Feminino } & \multicolumn{2}{c}{ Total } \\
& $\mathbf{n}$ & $\mathbf{\%}$ & $\mathbf{n}$ & $\mathbf{\%}$ & $\mathbf{n}$ & \% \\
\hline 6 anos & 149 & 51,7 & 159 & 48,0 & 308 & 49,8 \\
7 anos & 139 & 48,3 & 172 & 52,0 & 311 & 50,2 \\
6-7 anos & 288 & 46,5 & 331 & 53,5 & 619 & 100,0 \\
\hline
\end{tabular}

Quatro escolas em cada uma das seis regiões do município de Fortaleza foram selecionadas por conveniência para participar deste estudo, sendo duas escolas públicas e duas particulares, por região. Com a autorização da direção escolar, trinta alunos de cada escola foram selecionados aleatoriamente e convidados a participar da coleta dos dados. Dos 720 escolares convidados, $101(14,2 \%)$ crianças foram excluídas da amostra por não entregar o TCLE ou faltar o dia da coleta de dados. Portanto, a amostra deste estudo foi composta por 619 crianças, sendo 364 (59,9\%) de escolas públicas e $254(41,1 \%)$ de escolas particulares.

O critério utilizado para se determinar o intervalo entre as idades foi de 6,00-6,99 $=6$ anos e 7,00-7,99 $=7$ anos. A distribuição das crianças por idade e o sexo pode ser verificada na Tabela 1. 
Visando melhorar a confiabilidade das medidas, previamente à coleta de dados efetuouse um treinamento dos avaliadores. Os materiais utilizados para avaliação antropométrica foram: (i) fita métrica da marca Easyread e modelo Cateb, com resolução de $0,1 \mathrm{~cm}$; e (ii) balança digital da marca Plenna e modelo Wind, com resolução de $0,1 \mathrm{~kg}$ e capacidade de $150 \mathrm{~kg}$.

As padronizações propostas por Gordon, Chumlea e Roche (1988) foram seguidas para determinação da estatura $(\mathrm{m})$ e da massa corporal $(\mathrm{kg})$. A estatura foi aferida com a criança sem sapatos, os calcanhares unidos e a cabeça orientada com o plano de Frankfurt. Para determinação da massa corporal, a criança foi avaliada na posição ortostática, sem sapatos e usando o uniforme de educação física. As medidas da massa corporal e estatura foram utilizadas para cálculo do IMC $\left(\mathrm{kg} \cdot \mathrm{m}^{-2}\right)$.

O IMC foi classificado de acordo com os critérios propostos pela OMS (2007), Kuczmarski et al. (2002), Cole et al. $(2000,2007)$ e Conde e Monteiro (2006), a partir dos pontos de corte determinados para sexo e idade. O critério da OMS (2007) classificou os valores do IMC menores que o percentil 3 como desnutrição, entre os percentis 85 e 97 como sobrepeso e acima do percentil 97 como obesidade. O critério de Kuczmarski et al. (2002) classificou os valores do IMC menores que o percentil 5, entre 85 e 95,

\section{Resultados}

As características gerais da amostra de acordo com a idade e o sexo são apresentadas na Tabela 2. Observou-se diferença significativa entre os sexos para as variáveis massa corporal $(p=0,041)$ e estatura $(p=0,030)$ das crianças de seis anos de idade, sendo que os meninos apresentaram valores médios superiores.

Tabela 2. Média e desvio padrão das variáveis analisadas de acordo com a idade e sexo.

\begin{tabular}{lcccc}
\hline \multirow{2}{*}{ Variáveis } & \multicolumn{2}{c}{$\mathbf{6}$ anos } & \multicolumn{2}{c}{$\mathbf{7}$ anos } \\
\cline { 2 - 5 } & Masculino & Feminino & Masculino & Feminino \\
\hline Idade (anos) & $6,41(0,2)$ & $6,45(0,3)$ & $7,41(0,3)$ & $7,38(0,3)$ \\
Massa Corporal $(\mathrm{kg})$ & $23,6(5,0)$ & $22,5(4,5)^{\star}$ & $25,0(5,3)$ & $24,4(5,1)$ \\
Estatura $(\mathrm{cm})$ & $120,9(6,6)$ & $119,4(4,9)^{\star}$ & $124,5(6,1)$ & $123,6(6,2)$ \\
IMC $\left(\mathrm{kg} \cdot \mathrm{m}^{-2}\right)$ & $16,01(2,3)$ & $15,66(2,3)$ & $16,02(2,6)$ & $15,85(2,4)$ \\
\hline
\end{tabular}

* Diferença significativa, com $\mathrm{p}<0,05$.

$\mathrm{Na}$ Tabela 3, pode ser verificada a classificação do estado nutricional das crianças de acordo com os diferentes critérios de referência. Ao utilizar o critério da OMS (2007), não foram verificadas diferenças significativas entre os sexos nas classificações nutricionais $(p=0,308)$. Verificou-se também que o critério da OMS (2007) apresentou a maior prevalência de desnutrição $(16,0 \%)$ na amostra total e, ao utilizar e acima de 95 como desnutrição, sobrepeso e obesidade, respectivamente. Nos critérios de Cole et al. (2000) e Conde e Monteiro (2006) o sobrepeso e a obesidade foram identificados a partir das curvas equivalentes ao IMC $25 \mathrm{~kg} \cdot \mathrm{m}^{-2} \mathrm{e}$ $30,0 \mathrm{~kg} \cdot \mathrm{m}^{-2}$ para adultos, respectivamente. A desnutrição foi identificada à partir das curvas equivalentes ao IMC 18,5 kg.m ${ }^{-2}$ e $17,5 \mathrm{~kg} \cdot \mathrm{m}^{-2}$ para adultos de acordo com os critérios de et al. (2007) e Conde e Monteiro (2006), respectivamente. Os pontos de corte selecionados para a desnutrição, sobrepeso e obesidade corresponderam ao valor anual médio da idade dos escolares (ex. 6,5 anos).

A estatística descritiva foi utilizada para caracterização dos resultados, baseada em média, desvio padrão e porcentagem. Para verificar as diferenças entre os sexos utilizou-se o teste $t$ de Student para dados contínuos e o teste qui-quadrado $\left(X^{2}\right)$ para proporções. $O$ índice Kappa (k) foi utilizado para avaliar a concordância do critério da OMS (2007) com os demais critérios de classificação do estado nutricional. Utilizou-se o método proposto por Svanholm et al. (1989) para classificação da concordância em: pobre $(\leq 0,20)$, regular $(0,20$ a 0,40$)$, moderada $(0,41$ a 0,60$)$, boa $(0,61$ a 0,80$)$ e muito boa $(>0,80)$. O nível de significância foi estabelecido em $p<0,05$, utilizando-se o pacote estatístico SPSS 15.0 . o critério de Conde e Monteiro (2006), identificouse prevalência de desnutrição de apenas $4,4 \%$. Entretanto, no que se refere ao sobrepeso, percebeu-se que o critério brasileiro apresentou a maior prevalência (15,3\%), enquanto o critério de Kuczmarki et al. (2002) diagnosticou a menor proporção de crianças com sobrepeso $(10,5 \%)$. Os critérios da OMS (2007) e de Cole et al. $\underline{(2000,2007)}$ apresentaram a maior $(9,4 \%)$ e 
menor $(6,3 \%)$ prevalência de obesidade, respectivamente. De maneira geral, estes resultados também se repetiram ao utilizar os diferentes critérios de classificação em cada sexo.

Tabela 3. Classificação do estado nutricional dos escolares de Fortaleza, CE, a partir dos diferentes critérios de referência de acordo com o sexo.

\begin{tabular}{|c|c|c|c|c|}
\hline \multirow{2}{*}{ Critérios } & \multicolumn{4}{|c|}{ Estado Nutricional (\%) } \\
\hline & Desnutrição & Peso normal & Sobrepeso & Obesidade \\
\hline & \multicolumn{4}{|c|}{ Amostra total $(n=619)$} \\
\hline OMS (2007) & 16,0 & 62,4 & 12,3 & 9,4 \\
\hline Cole et al. $(2000,2007)$ & 15,3 & 67,2 & 11,1 & 6,3 \\
\hline Kuczmarski et al. (2002) & 10,0 & 70,3 & 10,5 & 9,2 \\
\hline \multirow[t]{2}{*}{ Conde e Monteiro (2006) } & 4,4 & 73,8 & 15,3 & 6,5 \\
\hline & \multicolumn{4}{|c|}{ Masculino $(n=288)^{*}$} \\
\hline OMS (2007) & 16,7 & 58,7 & 13,9 & 10,8 \\
\hline Cole et al. $(2000,2007)$ & 15,3 & 66,7 & 10,8 & 7,3 \\
\hline Kuczmarski et al. (2002) & 9,7 & 67,7 & 11,8 & 10,8 \\
\hline \multirow[t]{2}{*}{ Conde e Monteiro (2006) } & 3,1 & 74,0 & 17,4 & 5,5 \\
\hline & \multicolumn{4}{|c|}{ Feminino $(n=331)$} \\
\hline OMS (2007) & 15,4 & 65,6 & 10,9 & 8,2 \\
\hline Cole et al. $(2000,2007)$ & 15,4 & 67,7 & 11,5 & 5,4 \\
\hline Kuczmarski et al. (2002) & 10,3 & 72,5 & 9,4 & 7,9 \\
\hline Conde e Monteiro (2006) & 5,4 & 73,7 & 13,6 & 7,3 \\
\hline
\end{tabular}

${ }^{*}$ Não houve diferenças significativas entre os sexos, com $p<0,05$.

Os índices de concordância Kappa (k) do critério da OMS (2007) com os demais critérios de classificação do estado nutricional estão apresentados na Tabela 4. As concordâncias observadas foram significativas $(p<0,001)$, independente do sexo e idade. O critério de Kuczmarski et al. (2002) apresentou concordâncias muito boas em relação ao critério da OMS (2007), em ambos os sexos e idades e, enquanto que o critério de Cole et al. $(2000,2007)$ apresentou concordância muito boa para o sexo feminino e boa para o sexo masculino. Em relação à concordância do critério da OMS (2007) e de Conde e Monteiro (2006), observou-

se concordância moderada e boa, respectivamente, para as crianças do sexo masculino e feminino.

Quando analisada a amostra total, a menor concordância do critério da OMS (2007) foi verificada com o critério de $\underline{\text { Conde e Monteiro (2006) }}$ para o sexo masculino e a maior com o critério de Cole et al. (2000, 2007) para o sexo feminino.

Tabela 4. Índice de concordância Kappa (k) do critério da OMS (2007) com o critério de Kuczmarski et al. (2002), Cole et al. (2000, 2007) e Conde e Monteiro (2006), para os escolares de Fortaleza, CE, de acordo com a idade e sexo.

\begin{tabular}{|c|c|c|c|}
\hline & \multicolumn{3}{|c|}{ Critério OMS (2007)* } \\
\hline & $\begin{array}{c}\text { Kuczmarski et al. } \\
(2002)\end{array}$ & $\begin{array}{c}\text { Cole et al. } \\
(2000,2007)\end{array}$ & $\begin{array}{c}\text { Conde e Monteiro } \\
(2006)\end{array}$ \\
\hline \multicolumn{4}{|l|}{6 anos } \\
\hline Masculino $(n=149)$ & 0,80 & 0,76 & 0,58 \\
\hline Feminino $(n=159)$ & 0,85 & 0,92 & 0,78 \\
\hline Ambos $(n=308)$ & 0,83 & 0,84 & 0,68 \\
\hline \multicolumn{4}{|l|}{7 anos } \\
\hline Masculino $(n=139)$ & 0,80 & 0,76 & 0,65 \\
\hline Feminino $(n=172)$ & 0,81 & 0,85 & 0,70 \\
\hline Ambos $(n=311)$ & 0,80 & 0,81 & 0,68 \\
\hline \multicolumn{4}{|l|}{ 6-7 Anos } \\
\hline Masculino $(n=288)$ & 0,80 & 0,76 & 0,61 \\
\hline Feminino $(n=331)^{\prime}$ & 0,83 & 0,88 & 0,74 \\
\hline Ambos (619) & 0,82 & 0,82 & 0,68 \\
\hline
\end{tabular}

*Todas as concordâncias foram significativas, com $p<0,05$.

\section{Discussão}

Apesar da importância do diagnóstico do estado nutricional de crianças e adolescentes de diferentes regiões do Brasil, há poucos estudos a respeito do estado nutricional em crianças escolares do município de Fortaleza. No presente estudo, verificou-se que a desnutrição esteve 
mais presente do que o sobrepeso nas crianças deste município, entretanto, quando observadas as prevalências de sobrepeso e obesidade (excesso de peso), foi verificada uma maior prevalência em relação à desnutrição. Este perfil nutricional das crianças do município de Fortaleza ainda é observado em alguns municípios do Nordeste brasileiro, onde são identificadas elevadas prevalências de desnutrição, sobrepeso e obesidade no mesmo espaço sociodemográfico (IBGE, 2006).

Vale ressaltar que estes resultados não se justificam pela homogeneidade da amostra deste estudo, visto que foram avaliadas crianças tanto de escolas públicas quanto de particulares. Tampouco pelo critério de classificação do estado nutricional, uma vez que dos quatro critérios de classificação utilizados no presente estudo, três apresentaram prevalências semelhantes de desnutrição. Portanto, observa-se a necessidade de políticas públicas para combater tanto a condição de sobrepeso e obesidade quanto da desnutrição nas crianças do município de Fortaleza, CE.

Em relação aos critérios utilizados no presente estudo para comparação com o da OMS (2007), percebe-se que o critério proposto por $\underline{\text { Cole et al. }}$ $(2000,2007)$ é bastante utilizado no diagnóstico nutricional de crianças e adolescentes brasileiros (PELEGRINI et al., 2008; VIEIRA, et al., 2008), provavelmente por ter sido desenvolvido com base em dados de seis diferentes países, incluindo o Brasil. O critério de Kuczmarski et al. (2002) foi desenvolvido pelo Centers for Disease Control and Prevention dos Estados Unidos e também é bem aceito na classificação do estado nutricional de jovens brasileiros (BARRETO, 2007; PEREIRA; LIMA; MARTINS, 2004). No entanto, o critério de Conde e Monteiro (2006) ainda é pouco utilizado para classificação do estado nutricional em jovens, provavelmente devido à sua recentemente publicação. A acurácia deste critério para a classificação do estado nutricional tem sido investigada recentemente, principalmente por ser o único critério com dados de crianças e adolescentes brasileiros (FARIAS JÚNIOR et al., 2009; QUADROS, 2009; VITOLO et al., 2007; FERNANDES et al., 2007).

Ao analisar a concordância destes critérios de classificação do estado nutricional com o da $\underline{\mathrm{OMS}}$ (2007) em crianças, verificou-se que o recente critério da OMS apresentou concordâncias entre moderada e muito boa com os demais critérios investigados. Estes resultados corroboram outros estudos realizados com crianças brasileiras (LEAL, 2008; SILVA et al., 2008).

Os achados do presente estudo indicaram que o critério de Conde e Monteiro (2006) apresentou as menores concordâncias com o critério da OMS (2007), enquanto que o critério de Kuczmarski et al. (2002) foi o único que apresentou concordâncias muito boas em ambos os sexos e idades. Em contrapartida, Leal (2008) verificou que o critério de Conde e Monteiro (2006) apresentou maior concordância com o critério da OMS (2007) do que o critério de Cole et al. (2000, 2007) para ambos os sexos, ao estudar 2.795 crianças de 7 a 10 anos de idade do município de Florianópolis, SC. Vale ressaltar que Leal (2008) utilizou uma faixa etária diferente do presente estudo e analisou a concordância entre critérios somente para identificação do excesso de peso, o que pode justificar as discordâncias entre os resultados dos estudos.

As diferenças entre os critérios na concordância com o critério da OMS (2007) podem ser justificadas pelos pontos de corte do IMC de cada critério de classificação do estado nutricional. Alguns estudos já verificaram que o critério de Conde e Monteiro (2006) classifica mais casos de excesso de peso do que os critérios de Cole et al. $(2000,2007)$ e Kuczmarski et al. (2002), indicando que o critério brasileiro tende a superestimar a prevalência de excesso de peso quando comparado aos critérios norteamericano e internacional (FARIAS JÚNIOR et al., 2009; QUADROS, 2009; FERNANDES et al., 2007).

Estudo realizado com adolescentes de idades entre 10 e 19 anos indicou que a mudança do critério antigo da OMS (1995) para o atual (OMS, 2007) resultou em aumentos significativos nas prevalências de sobrepeso (GOMES; ANJOS; VASCONCELOS 2009). Além disso, a utilização do critério de Cole et al. $(2000,2007)$ resultou em uma redução significativa na prevalência de desnutrição quando comparado à recomendação atual da OMS (GOMES; ANJOS; VASCONCELOS 2009). Estes resultados sugerem cautela na comparação de estudos com diferentes critérios de classificação do estado nutricional, uma vez que cada critério possui particularidades, principalmente no que se refere 
á população de referência e ao método estatístico utilizado para estabelecimento dos pontos de corte.

A análise das concordâncias entre critérios para classificação do estado nutricional em crianças tem sido investigada com frequência na literatura (KO et al., 2008; WILLONS; JOHNSON; BALL, 2007; KAIN et al., 2002; FLEGAL et al., 2001). Entretanto, observa-se resultados destoantes entre os estudos, variando de concordâncias muito boas (LEITE et al. 2008) a pobres (FERNANDES et al., 2007; BUENO; FISBERG, 2006). Não obstante, pode-se verificar que muitos estudos avaliaram apenas a concordância entre critérios na detecção de excesso de peso em crianças e adolescentes (FARIAS JÚNIOR et al., 2009; FERNANDES et al., 2007; ABRANTES; LAMOUNIER; COLOSIMO, 2003). Contudo, no presente estudo optou-se por analisar a concordância dos critérios tanto na detecção do excesso de peso quanto da desnutrição, visto que a desnutrição ainda é presente em crianças de todas as regiões do Brasil, especialmente em algumas regiões do Nordeste brasileiro (IBGE, 2006), fato confirmado no presente estudo.

Vale destacar que poucos estudos foram encontrados na literatura analisando a concordância do critério da OMS (2007) com outros critérios de classificação do estado nutricional. A maioria dos estudos que utilizaram o critério da OMS foi realizada para analisar o padrão de crescimento em crianças menores de cinco anos de idade (ÁLVAREZ; LOPES; ESTRADA, 2009; LEONE; BERTOLI; SCHOEPS, 2009) ou para verificar a concordância deste critério com outros critérios de classificação do estado nutricional nesta faixa etária (MEl et al., 2008; PADULA; SALCEDA, 2008).

Com base neste contexto, a análise da concordância do critério da OMS (2007) com outros critérios utilizados na literatura brasileira foi relevante para verificar se o recente critério poderia ocasionar divergências com critérios anteriormente utilizados. Além disso, a avaliação do estado nutricional de crianças do município de Fortaleza, CE, a partir do critério da OMS (2007) foi coerente, uma vez que há evidências de que este critério segue um padrão metodológico mais adequado na determinação da desnutrição, sobrepeso ou obesidade (BORGHI et al., 2006). Contudo, cabe destacar que a amostra do presente estudo foi representativa de escolares de 6 e 7 anos de idade de um município do Nordeste brasileiro, fato que impossibilita a generalização dos resultados. Neste sentido, encoraja-se a realização de estudos que investiguem o estado nutricional em amostras que sejam representativas de outras idades e regiões do Brasil.

\section{Conclusão}

Pode-se concluir que a desnutrição, o sobrepeso e, em menor proporção, a obesidade, foram prevalentes em crianças de seis e sete anos de idade do município de Fortaleza, CE, de acordo com o recente critério de classificação do estado nutricional proposto pela OMS. Este critério apresentou concordâncias entre boa a muito boa com os critérios norte-americano e internacional e moderada a boa com o critério brasileiro. Portanto, sugere-se cautela na utilização do critério da OMS, assim como dos demais, para avaliação do estado nutricional em crianças brasileiras devido às diferenças étnicas e metodológicas.

\section{Referências}

ABRANTES, M.M.; LAMOUNIER, J.A.; COLOSIMO, E.A. Comparison of body mass index values proposed by Cole et al. (2000) and Must et al. (1991) for identifying children with weight-for-height index recommended by the World Health Organization. Public Health Nutrition, v.6, n.3, p.307-311, 2003. http://dx.doi.org/10.1079/PHN2002426

ÁLVAREZ, M.C.; LÓPEZ, A.; ESTRADA, A. Estado nutricional de niños de Antioquia, Colombia, según dos sistemas de referencia. Revista Panamericana de Salud Publica, v.25, n.3, p.196-203, 2009. Disponível em: www.scielosp.org/pdf/rpsp/v25n3/a02v25n3.pdf

\section{BARRETO, A.C.N.G. Prevalência de excesso de peso em pré-escolares na cidade de Natal/RN. 65f. Dissertação (Mestrado em Ciências da Saúde) Universidade Federal do Rio Grande do Norte, Natal, 2007.}

BATISTA FILHO, M.; RISSIN, A. A transição nutricional no Brasil: tendências regionais e temporais. Caderno de Saúde Pública, Rio de Janeiro,v.19, Supl.1,p.181-91, 2003.Disponível em:www.scielo.br/pdf/\%0D/csp/v19s1/a19v19s1.p df

BORGHI, E.; ONIS, M.; GARZA, C.; VAN DEN BROECK, J.; FRONGILLO, E.A.; GRUMMERSTRAWN, L.; VNA BUUREN, S.; MOLINARI, L.; 
MARTORELL, R.; ONYANGO, A.W.; MARTINES, J.C. Construction of the World Health

Organization child growth standards: selection of methods for attained growth curves. Statitics in Medicine, v.25, p.247-265, 2006.

http://dx.doi.org/10.1002/sim.2227

BUENO, M.B.; FISBERG, R.M. Comparação de três critérios de classificação de sobrepeso e obesidade entre pré-escolares. Revista Brasileira de Saúde Materno Infantil, Recife, v.6, n.4, p.411-417, 2006.

http://dx.doi.org/10.1590/S1519$\underline{38292006000400008}$

BUTTE N.F; GARZA C; ONIS, M. Evaluation of the feasibility of international growth standards for school-aged children and adolescents. The Journal of Nutrition, v.137, p.153-157, 2007.Disponível em:http://jn.nutrition.org/cgi/reprint /137/1/153

COLE, T.J. Development of an international growth standard for preadolescent and adolescent children: Statistical considerations. Food and Nutrition Bulletin, v.27, Supl.1, p.237-243, 2006.Disponível em:

http://www.unu.edu/ unupress/food/FNBv27n4 suppl 2 final.pdf

COLE, T.J.; BELLIZZI, M.C.; FLEGAL, K.M.; DIETZ, W.H. Establishing a standard definition for child overweight and obesity worldwide: international survey. British Medical Journal, v.320, n.7244, p.1240-3, 2000. Disponível em: http://www.ncbi.nlm.nih.gov/pmc/articles/PMC273 65/pdf/1240.pdf

COLE, T.J.; FLEGAL, K.M.; NICHOLLS, D.; JACKSON, A. Body mass index cut offs to define thinness in children and adolescents: international survey. British Medical Journal, v.335, n.7612, p.01-8, 2007. http://dx.doi.org/10.1136/bmi. $\underline{39238.399444 .55}$

COLE, T.J.; FREEMAN, J.V.; PREECE, M.A. Body mass index reference curves for the UK, 1990. Archives of Disease in Childhood, v.73, p.25-9, 1995. Disponível em:

http://www.ncbi.nlm.nih.gov/pmc/PMC1511150/

CONDE, W.L.; MONTEIRO, C.A. Body mass index cutoff points for evaluation of nutritional status in Brazilian children and adolescents. Jornal de Pediatria, Rio de Janeiro, v.82, n.4, p.266-272, 2006. Disponível em: www.scielo.br/pdf/jped/v82n4/v82n4a07.pdf

FARIAS JÚNIOR, J.C.; KONRAD, L.M.; RABACOW, F.M.; GRUP, S.; ARAÚJO, V.C. Sensibilidade e especificidade de critérios de classificação do índice de massa corporal em adolescentes. Revista de Saúde Pública, v.43, n.1, p.53-59, 2009.

http://dx.doi.org/10.1590/S003489102009000100007

FERNANDES, R.A.; ROSA, C.S.C.; SILVA, C.B.; BUENO, D.R.; OLIVEIRA, A.R.; FREITAS JÚNIOR, I.F. Desempenho de diferentes valores críticos de índice de massa corporal na identificação do excesso de gordura corporal e obesidade abdominal em adolescentes. Revista da Associação Médica Brasileira, v.53, n.6, p.515-9, 2007. http://dx.doi.org/10.1590/S0104$\underline{42302007000600019}$

FLEGAL, K. M.; OGDEN, C. L.; WEI, R.; KUCZMARSKI, R. L.; JOHNSON, C. L. Prevalence of overweight in US children: comparison of US growth charts from the Centers for Disease Control and Prevention with other reference values for body mass index. American Journal of Clinical Nutrition, v.73, n.6, p.108693, 2001.Disponível:

http://www.ajcn.org/cgi/reprint/73/6/1086?ck=nck

GOMES, F.S.; ANJOS, L.A.; VASCONCELOS, M.T.L. Influence of different body mass index cutoff values in assessing the nutritional status of adolescents in a household survey. Caderno de Saúde Pública, Rio de Janeiro, v.25, n.8, p.185057, 2009. http://dx.doi.org/10.1590/S0102311X2009000800021

GORDON, C. C.; CHUMLEA, W. C. C.; ROCHE, A. F. Stature, recumbent length, and weight. In: LOHMAN, T. G.; ROCHE, A. F.; MARTORELL, R. (ed.). Anthropometric standardization reference manual. Champaign, IL: Human Kinetics, 1988.

Instituto Brasileiro de Estatística e Geografia IBGE. Pesquisa Nacional por Amostra de Domicílios. Rio de Janeiro: IBGE; 2005; Disponível em: http://www.ibge.gov.br. Acesso em: 18 jan 2006.

Instituto Brasileiro de Estatística e Geografia IBGE. Pesquisa de Orçamentos Familiares (POF) 2002-2003. Disponível em:

http://www.ibge.gov.br. Acesso em: 23 jun. 2007.

JANSSEN, I.; KATZMARZYK, P.T.; SRINIVASAN, S.R.; CHEN, W.; MALINA, R.M.; BOUCHARD, C.; BERENSON, G.S. Utility of childhood BMI in the prediction of adulthood disease: comparison of national and international references. Obesity Research, v,13, p.1106-15, 2005. http://dx.doi.org/10.1038/oby.2005.129

KAIN, J.; UAUY, R.; VIO, F.; ALBALA, C. Trends in overweight and obesity prevalence in Chilean children: comparison of three definitions.

European Journal of Clinical Nutrition, v.56, p.200-4, 2002. Disponível em: 
http://www.nature.com/ejcn/journal/v56/n3/pdf/160 1301a.pdf

KATZMARZYK, P.T.; TREMBLAY, A.; PERUSSE, L.; DESPRES, J.; BOUCHARD, C. The utility of the international child and adolescent overweight guidelines for predicting coronary heart disease risk factors. Journal of Clinical Epidemiology, v.56, p.456-62, 2003.

http://dx.doi.org/10.1016/S0895-4356(02)00595-4

KO, G.T.C.; OZAKI, R.; WONG, G.W.K.; SO, W.Y.; TONG, P.; CHAN, M.; HO, C.S.; LAM, C.W.K.; CHAN, J.C.N. The problem of obesity among adolescents in Hong Kong: a comparison using various diagnostic criteria. BMC Pediatrics, v.8, n.10, p.1-8, 2008.

http://dx.doi.org/10.1186/1471-2431-8-10

Kuczmarski, R.J.; Ogden, C.L. Guo, S.S.; Grummer-Strawn, L.M.; FLEGAL, K.M.; MEI, Z.; WEI, R.; CURITN, L.R.; ROCHE, A.F. JOHNSON, C.L. 2000 CDC growth charts for the United States: methods and development. Vital Health Statistics, v.11, n.246, p.1-190, 2002. Disponível em:

http://www.ncbi.nlm.nih.gov/pubmed/12043359

LEAL, D.B. Sensibilidade e Especificidade dos sistemas de classificação para excesso de peso em crianças de 7-10 anos. 58f.

Dissertação (Mestrado em Educação Física).

Universidade Federal de Santa Catarina,

Florianópolis, 2008.

LEITE, N.; MILANO, G.E.; LOPES, W.A.; TANAKA, J.; DRESSLER, V.F.; RADOMINSKI, R.B. Comparação entre critérios para índice de massa corporal na avaliação nutricional em escolares. Revista da Educação Física, Maringá; v.19, n.4, p.557-63, 2008. http://dx.doi.org/10.4025/reveducfis.v19i4.4709

LEONE, C.; BERTOLI, C.J.; SCHOEPS, D.O. Novas curvas de crescimento da Organização Mundial da Saúde: comparação com valores de crescimento de crianças pré-escolares das cidades de Taubaté e Santo André, São Paulo. Revista Paulista de Pediatria, v.27, n.1, p.40-7, 2009. http://dx.doi.org/10.1590/S010305822009000100007

MAYNARD, L.M.; WISEMANDLE, W.; ROCHE, A.F.; CHUMLEA, W.C.; GUO, S.S.; SIERVOGEL, R.M. Childhood Body Composition in Relation to Body Mass Index. Pediatrics, v.107, n.2, p.34450, 2001. Disponível em:

http://pediatrics.aappublications.org/cgi/reprint/10 $\underline{7 / 2 / 344}$

MEI, Z.; OGDEN, C.L.; FLEGAL, K.M.; GRUMMER-STRAWN, L.M. Comparison of the Prevalence of Shortness, Underweight, and
Overweight among US Children Aged 0 to 59 Months by Using the CDC 2000 and the WHO 2006 Growth Charts. Journal of Pediatrics, v.153, p.622-8, 2008.

http://dx.doi.org/10.1016/j.jpeds.2008.05.048

MONTEIRO, C.A.; BENICIO, M.H.D.; KONNO, S.C.; SILVA, A.C.F.; et al. Causas do declínio da desnutrição infantil no Brasil, 1996-2007. Revista de Saúde Pública, São Paulo, v.43, n.1, p.35-43, 2009. http://dx.doi.org/10.1590/ S0034$\underline{89102009000100005}$

MONTEIRO, C.A.; CONDE, W.L.; POPKIN, B.M. The Burden of Disease From Undernutrition and Overnutrition in Countries Undergoing Rapid Nutrition Transition: A View From Brazil.

American Journal of Public Health, v.94, n.3, p.433-4, 2004. Disponível em:

http://www.ncbi.nlm.nih.gov/pmc/articles/ PMC1448269/

MUST, A.; DALLAL, G.E.; DIETZ, W.H. Reference data for obesity: 85 th and $95^{\text {th }}$ percentiles of body mass index (wt/ht ${ }^{2}$ ) and triceps skinfold thickhness. American Journal of Clinical Nutrition, v.53, n.4, p.839-46, 1991. Disponível em: http://www.ajcn.org/cgi/reprint/53/4/839

NEOVIUS, M.G.; LINNÉ, Y.M.; BARKELING, B.S.; ROSSNER, S.O. Sensitivity and specificity of classification systems for fatness in adolescents. American Journal of Clinical Nutrition, v.80, n.3, p.597-603, 2004. Disponível em: http://www.ajcn.org/cgi/reprint/80/3/597

NIHISER, A.J.; LEE, S.M.; WECHSLER, H.; MCKENNA, M.; ODOM, E.; REINOLD, C.; THOMPSON, D.; GRUMMER-STRAWN, L. Body mass index measurement in schools. Journal of School Health, v.77, p.651-71, 2007. Disponível em:

http://www.cdc.gov/healthyyouth/obesity/BMl/pdf/ BMI execsumm.pdf

ONIS, M.; ONYANGO, A.W.; BORGHI, E.; SIYAM, A.; NISHIDA, C. ; SIEKMANN, J. Development of a WHO growth reference for school-aged children and adolescents. Bulletin of World Health Organization, v.85, n.9, p.660-7, 2007.

Organização Mundial de Saúde - OMS. Physical status: the use and interpretation of anthropometry. Geneva: WHO, 1995. Disponível em:

http://www.unu.edu/unupress/food/FNBv27n4 su ppl 2 final.pdf

Organização Mundial de Saúde - OMS. Obesity: preventing and managing the global epidemic. Report of a WHO consultation, Geneva, 3-5 Jun 
1997. Geneva: World Health Organization, 1998. (WHO/NUT/98.1.)

Organização Mundial de Saúde - OMS. Growth reference data for 5-19 years. 2007. Disponível em:

http://www.who.int/growthref/who2007 bmi for a ge/en/index.html

PADULA, G.; SALCEDA, S.A. Comparación entre referencias de las prevalências de sobrepeso y obesidad, estimadas a través del Índice de Masa Corporal, en niños de Argentina. Arquivos

Latinoamericanos del Nutrición; v.58, n.4, p.330-5, 2008. Disponível em:

http://www.scielo.org.ve/pdf/alan/v58n4/art02.pdf

PELEGRINI, A.; PETROSKI, E.D.; COQUEIRO, R.S.; GAYA, A.C.A. Overweight and obesity in brazilian schoolchildren aged 10 to 15 years: data from a Brazilian sports Project. Arquivos

Latinoamericanos del Nutrición, v.58, n.4, p.343-9, 2008. Disponível em:http://www.scielo.org.ve/pdf/alan/v58n4/art04. pdf

PEREIRA, R.M.M.; LIMA, J.W.O.; MARTINS, M.C.V. Prevalência de sobrepeso e obesidade em estudantes de 7 a 14 anos em Escolas Públicas de Fortaleza-Ceará. Revista de Pediatria do Ceará, v.5, n.1, p.28-33, 2004. Disponível em:

http://www.socep.org.br/Rped/pdf/5.1\%20Art $\% 20$ Orig\%2002.pdf

QUADROS, T.M.B. Excesso de peso e adiposidade em crianças de 6 a 9 anos de idade. 125f. Dissertação (Mestrado em Educação Física) Universidade Federal de Santa Catarina, Florianópolis, 2009.

SILVA, H.G.; CHIARA, V.L.; BARROS, M.E.; RÊGO, A.L.; FERREIRA, A.; PITASI, B.A.; MATTOS, T. Diagnosing the nutritional status of schoolchildren: a comparison between Brazilian and international criteria. Jornal de Pediatria, Rio de Janeiro, v.84, n.6, p.550-5, 2008. http://dx.doi.org/10.1590/S002175572008000700013

SVANHOLM, H.; STARKLINT, H.; GUNDERSEN, H.J.; FABRICIUS, J.; BARLEBO, H.; OLSEN, S. Reproducibility of histomorphologic diagnoses with special reference to the Kappa statistic. APMIS; v.97, n.8, p.689-98, 1989. Disponível em: http://www.ncbi.nlm.nih.gov/pubmed/2669853

VIEIRA, M.F.A.; ARAÚJO, C.L.P.; HALLAL, P.C.; MADRUGA, S.W.; et al. Estado nutricional de escolares de 1a a 4a séries do Ensino Fundamental das escolas urbanas da cidade de Pelotas, Rio Grande do Sul, Brasil. Caderno de Saúde Pública, Rio de Janeiro, v.24, n.7, p.1667-
74, 2008. http://dx.doi.org/10.1590/S0102311X2008000700021

VITOLO, M. R.; CAMPAGNOLO, P. D. B.; BARROS, M. E.; GAMA, C. M.; LOPEZ, F. A. Avaliação de duas classificações para excesso de peso em adolescentes brasileiros. Revista de Saúde Pública, São Paulo, v.41, n.4, p.653-6, 2007. http://dx.doi.org/10.1590/S003489102007000400021

WANG, Y.; MONTEIRO, C.A.; POPKIN, B.M. Trends of obesity and underweight in older children and adolescents in the United States, Brazil, China and Russia. American Journal of Clinical Nutrition, v.75, n.6, p.971-977, 2002. Disponível em: http://www.ajcn.org/cgi/reprint/75/6/971

WILLOWS, N.D.; JOHNSON, M.; BALL, G.D.C. Prevalence estimates of overweight and obesity in cree preschool children in northern Quebec according to international and US reference criteria. American Journal of Public Health, v.97, p.311-6, 2007.

Valter Cordeiro Barbosa Filho

Rua Sanito Rocha, 135, ap.504, Bairro Cristo Rei Curitiba PR Brasil

80050-380

e-mail: valtercbf@gmail.com

Recebido em: 22 de maio de 2010.

Aceito em: 17 de outubro de 2010.

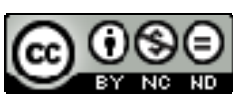

Motriz. Revista de Educação Física. UNESP, Rio Claro, SP, Brasil - elSSN: 1980-6574 - está licenciada sob Licença Creative Commons 